\title{
Bound states in weakly deformed strips and layers
}

\author{
D. Borisov, P. Exner, R. Gadyl'shin, and D. Krejčiřík
}

\begin{abstract}
We consider Dirichlet Laplacians on straight strips in $\mathbb{R}^{2}$ or layers in $\mathbb{R}^{3}$ with a weak local deformation. First we generalize a result of Bulla et al. to the three-dimensional situation showing that weakly coupled bound states exist if the volume change induced by the deformation is positive; we also derive the leading order of the weak-coupling asymptotics. With the knowledge of the eigenvalue analytic properties, we demonstrate then an alternative method which makes it possible to evaluate the next term in the asymptotic expansion for both the strips and layers. It gives, in particular, a criterion for the bound-state existence in the critical case when the added volume is zero.
\end{abstract}

\section{Introduction}

Spectra of Dirichlet Laplacians in infinitely stretched regions such as a planar strip or a layer of a fixed width have attracted a lot of attention recently. Of course, the problem is trivial as long as the strip or layer is straight because then one can employ separation of variables. However, already a local perturbation such as bending, deformation, or a change of boundary conditions can produce a non-empty discrete spectrum.

This effect was studied intensively in the last decade, first because it had applications in condensed matter physics, and also because it was itself an interesting mathematical problem. A particular aspect 
we will be concerned with here is the behaviour in the weak-coupling regime, i.e. the situation when the perturbation is gentle.

Recall that the answer to this question depends on the type of the perturbation. For bend strips, e.g., one can perform the BirmanSchwinger analysis which yields the first term in the asymptotic expansion for the gap between the eigenvalue and the threshold of the essential spectrum [DE]. It is proportional to the fourth power of the bending angle and always positive, since any nontrivial (local) bending induces a non-empty discrete spectrum. A local switch of the boundary condition from Dirichlet to Neumann has a similar effect. Here the weak-coupling behaviour was determine variationally to be governed by the fourth power of the "window width" [EV1] and the exact asymptotics was derived formally in $[\mathrm{PB}]$ by a direct application of the technique developed in [1], Ga]. Notice that this asymptotics differs substantially from that corresponding to a local change in the mixed boundary conditions, where the Birman-Schwinger technique is applicable and the leading term is a multiple of the square of the said parameter EK]. Recall also that analogous results can be derived for layers with locally perturbed boundary conditions where, however, the asymptotics is exponential rather that powerlike EV2.

The present paper deals with the case of a local deformation of the strip or layer, which is more subtle than the bending or boundarycondition modification. The main difference that the effective interaction induced by a deformation can be of different signs, both attractive and repulsive. It is easy to see by bracketing that a bulge on a strip or layer does create bound states while a squeeze does not. The answer is less clear for more complicated deformations where the width change does not have a definite sign.

The first rigorous treatment of this problem was presented in the work of Bulla et al [BGRS] dealing with a local one-sided deformation (characterized by a function $\lambda v$ ) of a straight strip of a constant width $d$. The authors found that the added volume was decisive: a bound state exists for small positive $\lambda$ if the area change $\lambda d\langle v\rangle$ is positive, and in that case the ground-state eigenvalue has the following 
weak-coupling expansion,

$$
E(\lambda)=\kappa_{1}^{2}-\lambda^{2} \kappa_{1}^{4}\langle v\rangle^{2}+\mathcal{O}\left(\lambda^{3}\right),
$$

where $\kappa_{1}=\frac{\pi}{d}$ is the square root of the first transverse eigenvalue. 10 On the other hand, the discrete spectrum is empty if $\langle v\rangle<0$. A problem arises in the critical case, $\langle v\rangle=0$, when the areas of the outward and inward deformation coincide. The authors of [BGRS suggested that the analogy with one-dimensional Schrödinger operators by which bound states should exist again may be misleading due to the presence of the higher transverse modes.

This suspicion was confirmed in $[E V 3]$ where it was shown that this is true only if the deformation was "smeared" enough. More specifically, the discrete spectrum is empty if

$$
d>\frac{4}{\sqrt{3}} b
$$

provided $\operatorname{supp} v \subset[-b, b]$. On the other hand, a weakly bound state exists if

$$
\frac{\left\|v^{\prime}\right\|^{2}}{\|v\|^{2}}<\frac{6 \kappa_{1}^{2}}{9+\sqrt{90+12 \pi^{2}}}
$$

and in that case there are positive $c_{1}, c_{2}$ such that

$$
-c_{1} \lambda^{4} \leq E(\lambda)-\kappa_{1}^{2} \leq-c_{2} \lambda^{4} \text {. }
$$

These results have been obtained by a variational method and they are certainly not optimal, because there are deformed strips which fulfill neither of the conditions (1.2), (1.3).

A way to improve the above conclusions would be to compute the Birman-Schwinger expansion employed in [BGRS] to the second order which becomes the leading one when the term linear in $\lambda^{2}$ in (1.1) is absent, and the asymptotics is governed by $\lambda^{4}$ in correspondence with (1.4). This is not easy, however. The standard technique in these

\footnotetext{
${ }^{1}$ In fact, they assumed $d=1$, but it is easy to restore the strip width in their expression obtaining eq. (1.1).
} 
situations is to map the strip in question onto a straight one by means of suitable curvilinear coordinates. In distinction to the bent-strip case [DE] these coordinates typically are not locally orthogonal. Hence the transformed Laplacian contains numerous terms which make the computation extremely cumbersome.

After this introduction, let us describe the aim and the scope of the present paper. The aim is twofold. First we are going to consider an extension of the result of BGRS to the case of a locally deformed layer. The result is summarized in Theorem 2.4. In particular, we derive a weak-coupling expansion of the ground-state eigenvalue,

$$
E(\lambda)=\kappa_{1}^{2}-\exp \left[2\left(-\lambda \frac{\kappa_{1}^{2}}{\pi}\langle v\rangle+\mathcal{O}\left(\lambda^{2}\right)\right)^{-1}\right]
$$

and show the analytical properties of the round-bracket expression w.r.t. $\lambda$. This is done in Sec. 2 ; the results again say nothing about the behaviour in the critical case.

Instead of attempting to proceed further by the Birman-Schwinger method, we demonstrate in Sec. 3 a different approach to the weakcoupling problem. It is based on constructing the asymptotics of a particular boundary value problem, and requires as a prerequisite the analyticity of the function $E(\cdot)$ itself in dimension two, and of its above mentioned constituent in dimension three. In the present case, however, these properties are guaranteed by [BGRS] and the results of Sec. 2. The methods allows us to recover the expansions (1.1) and (1.5) in a different way. What is more, we are also able to compute higher terms, in principle of any order. We perform the explicit computation for the second-order terms which play role in the critical case. In particular, we made in this way more precise the result expressed by (1.2) and (1.3) about the critical bound-state existence for smeared perturbations, and derive its analog in the deformed-layer case.

\section{Locally deformed layers}




\subsection{The curvilinear coordinates}

Let $x=\left(x^{1}, x^{2}\right) \in \mathbb{R}^{2}$ and $(x, u) \in \Omega_{0}:=\mathbb{R}^{2} \times(0, d)$ with $d>0$. Given a function $v \in \boldsymbol{C}_{0}^{\infty}\left(\mathbb{R}^{2}\right)$ we define the mapping $(\lambda>0)$

$$
\phi: \Omega_{0} \rightarrow \mathbb{R}^{3}:\left\{(x, u) \mapsto \phi(x, u):=\left(x^{1}, x^{2},(1+\lambda v(x)) u\right)\right\}
$$

for $\lambda>0$, which defines our deformed layer $\Omega_{\lambda}:=\phi\left(\Omega_{0}\right)$.

To make use of the curvilinear coordinates defined by the mapping $\phi$ we need the metric tensor $G_{i j}:=\phi_{, i} . \phi_{, j}$ of the deformed layer. It can be seen easily to be of the form

$$
\left(G_{i j}\right)=\left(\begin{array}{ccc}
1+\lambda^{2} v_{, 1}^{2} u^{2} & \lambda^{2} v_{, 1} v_{, 2} u^{2} & \lambda v_{, 1}(1+\lambda v) u \\
\lambda^{2} v_{, 1} v_{, 2} u^{2} & 1+\lambda^{2} v_{, 2}^{2} u^{2} & \lambda v_{, 2}(1+\lambda v) u \\
\lambda v_{, 1}(1+\lambda v) u & \lambda v_{, 2}(1+\lambda v) u & (1+\lambda v)^{2}
\end{array}\right)
$$

where $v_{, \mu}$ means the derivative w.r.t. $x^{\mu}$, and its determinant is $G:=$ $\operatorname{det}\left(G_{i j}\right)=(1+\lambda v)^{2}$.

In view of the inverse function theorem, the mapping $\phi$ defining the layer will be diffeomorphism provided $\lambda\left\|v_{-}\right\|_{\infty}<1$, where we put conventionally $v_{-}:=\max \{0,-v\}$. For a sign-changing $v$, this is a nontrivial restriction which is satisfied, however, when $\lambda$ is small enough. That is just the case we are interested in.

We will also need the contravariant metric tensor, in other words the inverse matrix

$$
\left(G^{i j}\right)=\left(\begin{array}{ccc}
1 & 0 & -\frac{\lambda v, 1 u}{1+\lambda v} \\
0 & 1 & -\frac{\lambda v, 2 u}{1+\lambda v} \\
-\frac{\lambda v, 1 u}{1+\lambda v} & -\frac{\lambda v, 2 u}{1+\lambda v} & \frac{1+\lambda^{2} \mid \nabla v{ }^{2} u^{2}}{(1+\lambda v)^{2}}
\end{array}\right)
$$

and the following contraction identities

$$
G_{, j}^{\mu j}=-\frac{\lambda v_{, \mu}}{1+\lambda v}, \quad G_{, j}^{3 j}=-\frac{\lambda \Delta v u}{1+\lambda v}+\frac{3 \lambda^{2}|\nabla v|^{2} u}{(1+\lambda v)^{2}},
$$

where conventionally summation is performed over repeated indices, and we denote $|\nabla v|^{2}:=v_{, 1}^{2}+v_{, 2}^{2}$ and $\Delta v:=v_{, 11}+v_{, 22}$. Another convention concerns the range of the indices, which is 1,2 for Greek and 1,2,3 for Latin indices. The indices are at that associated with the above coordinates by $(1,2,3) \leftrightarrow\left(x^{1}, x^{2}, u\right)$. 


\subsection{The straightening transformation}

As mentioned in the introduction the main object of our study is the Dirichlet Laplacian $-\Delta_{D}^{\Omega_{\lambda}}$ on $\boldsymbol{L}^{2}\left(\Omega_{\lambda}\right)$. If we think of a quantum particle living in the region $\Omega_{\lambda}$ with hard walls and exposed to no other interaction, $-\Delta_{D}^{\Omega_{\lambda}}$ will be its Hamiltonian up to a multiplicative constant; we can get rid of the latter by setting the Planck's constant $\hbar=1$ and the effective mass $m_{*}=\frac{1}{2}$. Mathematically speaking, $-\Delta_{D}^{\Omega_{\lambda}}$ is defined for an open set $\Omega_{\lambda} \subset \mathbb{R}^{3}$ as the Friedrichs extension of the free Laplacian with the domain $\boldsymbol{C}_{0}^{\infty}(\Omega)-c f$. [RS, Sec. XIII.15]. Moreover, since the smooth boundary of $\Omega_{\lambda}$ has the segment property, $-\Delta_{D}^{\Omega_{\lambda}}$ acts simply as $\psi \mapsto-\psi_{, j j}$ with the Dirichlet b.c. at $\partial \Omega_{\lambda}$.

A natural way to investigate the Hamiltonian is to introduce the unitary transformation $U: \boldsymbol{L}^{2}\left(\Omega_{\lambda}\right) \rightarrow \boldsymbol{L}^{2}\left(\Omega_{0}\right):\left\{\psi \mapsto U \psi:=G^{\frac{1}{4}} \psi \circ \phi\right\}$ and to investigate the unitarily equivalent operator

$$
H_{\lambda}:=U\left(-\Delta_{D}^{\Omega_{\lambda}}\right) U^{-1}=-G^{-\frac{1}{4}} \partial_{i} G^{\frac{1}{2}} G^{i j} \partial_{j} G^{-\frac{1}{4}}
$$

with the form domain $Q\left(H_{\lambda}\right)=\boldsymbol{W}_{0}^{1,2}\left(\Omega_{0}\right)$ instead of $-\Delta_{D}^{\Omega_{\lambda}}$. As usual in such situations, the "straightened" region is geometrically simpler and the price we pay is a more complicated form of the operator (2.5).

To make it more explicit, put $F:=\ln G^{\frac{1}{4}}$. Commuting $G^{-\frac{1}{4}}$ with the gradient components, we cast the operator (2.5) into a form which has a simpler kinetic part,

$$
H_{\lambda}=-\partial_{i} G^{i j} \partial_{j}+V=-G^{i j} \partial_{i} \partial_{j}-G_{, j}^{i j} \partial_{i}+V,
$$

but contains an effective potential,

$$
V:=\left(G^{i j} F_{, j}\right)_{, i}+F_{, i} G^{i j} F_{, j}=G^{i j} F_{, i j}+G_{, j}^{i j} F_{, i}+G^{i j} F_{, i} F_{, j} .
$$

If we now employ the particular form (2.2) of the metric tensor together with (2.3), (2.4), we can write

$$
\begin{aligned}
H_{\lambda}= & -\partial_{1}^{2}-\partial_{2}^{2}-\frac{1+\lambda^{2}|\nabla v|^{2} u^{2}}{(1+\lambda v)^{2}} \partial_{3}^{2}+\frac{2 \lambda v_{, 1} u}{1+\lambda v} \partial_{1} \partial_{3}+\frac{2 \lambda v_{, 2} u}{1+\lambda v} \partial_{2} \partial_{3} \\
& +\frac{\lambda v, 1}{1+\lambda v} \partial_{1}+\frac{\lambda v_{, 2}}{1+\lambda v} \partial_{2}+\left(\frac{\lambda \Delta v u}{1+\lambda v}-\frac{3 \lambda^{2}|\nabla v|^{2} u}{(1+\lambda v)^{2}}\right) \partial_{3}+V
\end{aligned}
$$


with

$$
V=\frac{\lambda \Delta v}{2}-\frac{\lambda^{2} v \Delta v}{2(1+\lambda v)}-\frac{3 \lambda^{2}|\nabla v|^{2}}{4(1+\lambda v)^{2}} .
$$

For our purpose it useful to rewrite this expression further in a form sorted w.r.t. to the powers of $\lambda$ :

$$
\begin{aligned}
H_{\lambda}=- & \Delta_{D}^{\Omega_{0}}+\lambda\left[2 v \partial_{3}^{2}+2 v_{, 1} u \partial_{1} \partial_{3}+2 v_{, 2} u \partial_{2} \partial_{3}+v_{, 1} \partial_{1}+v_{, 2} \partial_{2}\right. \\
& \left.+(\Delta v) u \partial_{3}+\frac{\Delta v}{2}\right] \\
-\lambda^{2}[ & {\left[\frac{3 v^{2}+|\nabla v|^{2} u^{2}+2 \lambda v^{3}}{(1+\lambda v)^{2}} \partial_{3}^{2}+\frac{2 v v_{, 1} u}{1+\lambda v} \partial_{1} \partial_{3}+\frac{2 v v{ }_{, 2} u}{1+\lambda v} \partial_{2} \partial_{3}\right.} \\
& +\frac{v v_{, 1}}{1+\lambda v} \partial_{1}+\frac{v v_{, 2}}{1+\lambda v} \partial_{2}+\left(\frac{v(\Delta v) u}{1+\lambda v}+\frac{3|\nabla v|^{2} u}{(1+\lambda v)^{2}}\right) \partial_{3} \\
& \left.+\frac{v \Delta v}{2(1+\lambda v)}+\frac{3|\nabla v|^{2}}{4(1+\lambda v)^{2}}\right]
\end{aligned}
$$

In analogy with [BGRS], we thus get the following formula for the "straightened" operator,

$$
H_{\lambda}=H_{0}+\lambda \sum_{n=1}^{3} A_{n}^{*} B_{n}+\lambda^{2} \sum_{n=4}^{7} A_{n}^{*} B_{n},
$$

where each of the $A_{n}$ 's and $B_{n}$ 's is a first-order differential operator 
with compactly supported coefficients and

$$
\begin{array}{lll}
A_{1}^{*}:=2 v \partial_{3} & B_{1}:=\omega \partial_{3} \\
A_{2}^{*}:=\Delta v & B_{2}:=\omega\left(u \partial_{3}+\frac{1}{2}\right) \\
A_{3}^{*}:=\left(2 u \partial_{3}+1\right) \omega & B_{3}:=v_{, 1} \partial_{1}+v_{, 2} \partial_{2} \\
A_{4}^{*}:=-\frac{3 v^{2}+|\nabla v|^{2} u^{2}+2 \lambda v^{3}}{\left(1+\lambda v^{3}\right)} \partial_{3} & B_{4}:=\omega \partial_{3} \\
A_{5}^{*}:=-\frac{v \Delta v}{1+\lambda v} & B_{5}:=\omega\left(u \partial_{3}+\frac{1}{2}\right) \\
A_{6}^{*}:=-\frac{3|\nabla v|^{2}}{(1+\lambda v)^{2}} & B_{6}:=\omega\left(u \partial_{3}+\frac{1}{4}\right) \\
A_{7}^{*}:=-\frac{2 u \partial_{3}+1}{1+\lambda v} v & B_{7}:=v{ }_{, 1} \partial_{1}+v_{, 2} \partial_{2}
\end{array}
$$

with $\omega \in C_{0}^{\infty}\left(\mathbb{R}^{2}\right)$ such that $\omega \equiv 1$ on $\operatorname{supp} v$. We define a pair of operators $C_{\lambda}, D: \boldsymbol{L}^{2}\left(\Omega_{0}\right) \rightarrow \boldsymbol{L}^{2}\left(\Omega_{0}\right) \otimes \mathbb{C}^{7}$ by

$$
\begin{aligned}
& \varphi \mapsto\left(C_{\lambda} \varphi\right)_{n}:=\left\{\begin{array}{rr}
A_{n} \varphi & n=1,2,3 \\
\lambda A_{n} \varphi & n=4, \ldots, 7
\end{array}\right. \\
& \varphi \mapsto(D \varphi)_{n}:=B_{n} \varphi \quad n=1, \ldots, 7
\end{aligned}
$$

then (2.6) finally becomes $H_{\lambda}=H_{0}+\lambda C_{\lambda}^{*} D$.

\subsection{Weak coupling analysis}

First we note that since the our layer is deformed only locally, we have

$$
\sigma_{\mathrm{ess}}\left(-\Delta_{D}^{\Omega_{\lambda}}\right)=\sigma_{\mathrm{ess}}\left(-\Delta_{D}^{\Omega_{0}}\right)=\left[\kappa_{1}^{2}, \infty\right) .
$$

This is easy to see, for instance, by using a bracketing to show that $\inf \sigma_{\text {ess }}\left(-\Delta_{D}^{\Omega_{\lambda}}\right)=\kappa_{1}^{2}-c f$. DEK - while the opposite inclusion is obtained by constructing an appropriate Weyl sequence. We use the notation $\kappa_{j}^{2}:=\left(\frac{\pi}{d} j\right)^{2}$ for the eigenvalues of the transverse operator $\left(-\partial_{3}^{2}\right)^{D}$; the corresponding eigenfunctions are denoted by $\chi_{j}$, and 
their explicit form is

$$
\chi_{j}(u)=\sqrt{\frac{2}{d}} \sin \kappa_{n} u .
$$

Next we define $K_{\lambda}^{\alpha}:=\lambda D\left(H_{0}-\alpha^{2}\right)^{-1} C_{\lambda}^{*}$. We are interested in (positive) eigenvalues $E(\lambda)=: \alpha^{2}$ of $H_{\lambda}$ below the lowest transverse mode, hence we choose $\alpha \in\left[0, \kappa_{1}\right)$. Our basic tool is the following classical result - cf. [BGRS, Lemma 2.1]:

\section{Proposition 2.1 (Birman-Schwinger principle)}

$$
\alpha^{2} \in \sigma_{\text {disc }}\left(H_{\lambda}\right) \Longleftrightarrow-1 \in \sigma_{\text {disc }}\left(K_{\lambda}^{\alpha}\right)
$$

ProOF: If $K_{\lambda}^{\alpha} \psi=-\psi$, then $\varphi:=-\lambda\left(H_{0}-\alpha^{2}\right)^{-1} C_{\lambda}^{*} \psi$ is easily checked to satisfy $H_{\lambda} \varphi=\alpha^{2} \varphi$. Conversely, if $H_{\lambda} \varphi=\alpha^{2} \varphi$, we have $\varphi \in Q\left(H_{\lambda}\right) \subset D(D)$, so $\psi:=D \varphi$ is in $\boldsymbol{L}^{2}\left(\Omega_{0}\right)$ and $K_{\lambda}^{\alpha} \psi=-\psi$.

To make use of the above equivalence, we have to analyze the structure of $K_{\lambda}^{\alpha}$. Let $R_{0}(\alpha):=\left(H_{0}-\alpha^{2}\right)^{-1}$ be the free resolvent corresponding to $H_{0}$. Using the transverse-mode decomposition and the fact that $H_{0}=-\Delta^{\mathbb{R}^{2}} \otimes I_{1}+I_{2} \otimes\left(-\partial_{3}^{2}\right)^{D}$, we can express the integral kernel of $R_{0}$,

$$
R_{0}\left(x, u, x^{\prime}, u^{\prime} ; \alpha\right)=\sum_{j=1}^{\infty} \chi_{j}(u) r_{j}\left(x, x^{\prime} ; \alpha\right) \chi_{j}\left(u^{\prime}\right)
$$

where $r_{j}\left(x, x^{\prime} ; \alpha\right)$ is the kernel of $\left(-\Delta^{2}+\kappa_{j}^{2}-\alpha^{2}\right)^{-1}$ in $\boldsymbol{L}^{2}\left(\mathbb{R}^{2}\right)$. We define $k_{j}(\alpha)^{2}:=\kappa_{j}^{2}-\alpha^{2}$. The free kernel $r_{j}$ can be expressed in terms of Hankel's functions - cf. [AGH, Chap. I.5] - which are related to Macdonald's functions by [AS, 9.6.4], so finally we arrive at the formula

$$
R_{0}\left(x, u, x^{\prime}, u^{\prime} ; \alpha\right)=\frac{1}{2 \pi} \sum_{j=1}^{\infty} \chi_{j}(u) K_{0}\left(k_{j}(\alpha)\left|x-x^{\prime}\right|\right) \chi_{j}\left(u^{\prime}\right)
$$

Now we want to split the singular part of $R_{0}^{\alpha}$; we write $K_{\lambda}^{\alpha}=\hat{L}_{\lambda}+\hat{M}_{\lambda}$ where $\hat{L}_{\lambda}:=\lambda D L_{\alpha} C_{\lambda}^{*}$ contains the singularity:

$$
L_{\alpha}\left(x, u, x^{\prime}, u^{\prime}\right):=-\frac{1}{2 \pi} \chi_{1}(u) \ln k_{1}(\alpha) \chi_{1}\left(u^{\prime}\right)
$$


diverges logarithmically as $\alpha \rightarrow \kappa_{1}-$. The regular part $\hat{M}_{\lambda}=\lambda D M_{\alpha} C_{\lambda}^{*}$ consists of two terms, $M_{\alpha}=N_{\alpha}+R_{0}^{\perp}(\alpha)$, where the operator $R_{0}^{\perp}$ is defined as the projection of the resolvent on higher transverse modes

$$
R_{0}^{\perp}\left(x, u, x^{\prime}, u^{\prime} ; \alpha\right):=\frac{1}{2 \pi} \sum_{j=2}^{\infty} \chi_{j}(u) K_{0}\left(k_{j}(\alpha)\left|x-x^{\prime}\right|\right) \chi_{j}\left(u^{\prime}\right),
$$

and the remaining term is therefore

$$
N_{\alpha}\left(x, u, x^{\prime}, u^{\prime}\right):=\frac{1}{2 \pi} \chi_{1}(u)\left(K_{0}\left(k_{1}(\alpha)\left|x-x^{\prime}\right|\right)+\ln k_{1}(\alpha)\right) \chi_{1}\left(u^{\prime}\right) .
$$

Put $w^{-1}:=\ln k_{1}(\alpha)$. The next step in the BS method is to show the boundedness and the analyticity (w.r.t. $w$ ) of the regular part of $K_{\lambda}^{\alpha}$. A more difficult part of this task concerns the operator containing $N_{\alpha}$ where we have to take a different route than that used in [BGRS].

First we note that while the Hilbert-Schmidt norm is suitable for estimating the operator $N_{\alpha}$, it fails when the latter is sandwiched between $\lambda D$ and $C_{\lambda}^{*}$. More specifically, using the regularity and compact support of the functions involved one could transform $\lambda D N_{\alpha} C_{\lambda}^{*}$ into an integral operator via integration by parts, but the obtained kernel has a singularity which is not square integrable. Hence we use instead the "continuous" version of the Schur-Holmgren bound. Since it seems to be less known than its discrete analogue AGH, Lemma C.3], [Mad, Thm. 7.1.9], we present it here with the proof.

Lemma 2.2 Suppose that $M$ is an open subset of $\mathbb{R}^{n}$ and let $K$ : $\boldsymbol{L}^{2}(M) \rightarrow \boldsymbol{L}^{2}(M)$ be an integral operator with the kernel $K(\cdot, \cdot)$. Then

$$
\|K\| \leq\|K\|_{\mathrm{SH}}:=\left(\sup _{x \in M} \int_{M}\left|K\left(x, x^{\prime}\right)\right| d x^{\prime} \sup _{x^{\prime} \in M} \int_{M}\left|K\left(x, x^{\prime}\right)\right| d x\right)^{\frac{1}{2}} .
$$

Proof: The claim follows from the inequality

$$
\|K\|_{p, p} \leq\|K\|_{1,1}^{1 / p}\|K\|_{\infty, \infty}^{1 / q},
$$

where $K$ is now an integral operator on $\boldsymbol{L}^{p}(M), p^{-1}+q^{-1}=1$, and

$$
\|K\|_{\infty, \infty}:=\sup _{x \in M} \int_{M}\left|K\left(x, x^{\prime}\right)\right| d x^{\prime}, \quad\|K\|_{1,1}:=\sup _{x^{\prime} \in M} \int_{M}\left|K\left(x, x^{\prime}\right)\right| d x .
$$


If $K$ is bounded for $p=1, \infty$, we can prove (2.7) for the other $p$ by an interpolation argument adapted from the discrete case [Mad. By Hölder's inequality

$$
\begin{gathered}
\left|\int_{M} K\left(x, x^{\prime}\right) \psi\left(x^{\prime}\right) d x^{\prime}\right| \leq \int_{M}\left|K\left(x, x^{\prime}\right)\right|^{\frac{1}{p}}\left|K\left(x, x^{\prime}\right)\right|^{\frac{1}{q}}\left|\psi\left(x^{\prime}\right)\right| d x^{\prime} \\
\leq\left(\int_{M}\left|K\left(x, x^{\prime}\right)\right|\left|\psi\left(x^{\prime}\right)\right|^{p} d x^{\prime}\right)^{\frac{1}{p}} \int_{M}\left|K\left(x, x^{\prime}\right)\right| d x^{\prime}
\end{gathered}
$$

so we can easily estimate the $\boldsymbol{L}^{p}$-norm of $K \psi$,

$$
\begin{aligned}
\|K \psi\|_{p}^{p} & =\int_{M} d x\left|\int_{M} K\left(x, x^{\prime}\right) \psi\left(x^{\prime}\right) d x^{\prime}\right|^{p} \\
& \leq\|K\|_{\infty, \infty}^{p / q} \int_{M} d x \int_{M}\left|K\left(x, x^{\prime}\right) \| \psi\left(x^{\prime}\right)\right|^{p} d x^{\prime} \\
& \leq\|K\|_{\infty, \infty}^{p / q} \int_{M} d x^{\prime}\left|\psi\left(x^{\prime}\right)\right|^{p} \int_{M} d x\left|K\left(x, x^{\prime}\right)\right| \\
& \leq\|K\|_{\infty, \infty}^{p / q}\|K\|_{1,1}\|\psi\|_{p}^{p},
\end{aligned}
$$

which yields the result.

Recall that $\|\cdot\|_{\mathrm{SH}}$ is not a norm and that it simplifies for the symmetric kernels, $\|K\|_{\mathrm{SH}}=\sup _{x \in M} \int_{M}\left|K\left(x, x^{\prime}\right)\right| d x^{\prime}$. We are now ready to prove the following key result.

Lemma 2.3 $w \mapsto \hat{M}(\alpha(w))$ is a bounded and analytic operator-valued function, which can be continued from $\{w \in \mathbb{C} \mid \operatorname{Re} w<0\}$ to a region that includes $w=0$.

Proof: As in BGRS, Lemma 2.2], let $\mathcal{H}_{1} \subset \boldsymbol{L}^{2}\left(\Omega_{0}\right)$ be the space of $\boldsymbol{L}^{2}\left(\Omega_{0}\right)$ functions of the form $\varphi \chi_{1}$, where $\varphi \in \boldsymbol{L}^{2}\left(\mathbb{R}^{2}\right)$. Let further $\mathcal{P}_{1}$ be the projection onto this subspace, and $\mathcal{P}_{1}^{\perp}:=I-\mathcal{P}_{1}$ the projection onto its orthogonal complement in $\boldsymbol{L}^{2}\left(\Omega_{0}\right)$. Then $R_{0}^{\perp}(\alpha) \equiv R_{0}(\alpha) \mathcal{P}_{1}^{\perp}$ has an analytic continuation into the region $\left\{\alpha \in \mathbb{C} \mid \alpha^{2} \in \mathbb{C} \backslash\left[3 \kappa_{1}^{2}, \infty\right)\right\}$ since the lowest point in the spectrum of $H_{0} \mathcal{P}_{1}^{\perp} \uparrow \mathcal{P}_{1}^{\perp} \boldsymbol{L}^{2}\left(\Omega_{0}\right)$ is $\kappa_{2}^{2}-\kappa_{1}^{2}$. This region includes the domain $\left[0, \kappa_{1}\right)$ actually considered. To accommodate the extra factors $D, C_{\lambda}^{*}$, we introduce the quadratic form

$$
b_{\alpha}(\phi, \psi):=\left(\phi, D R_{0}^{\perp}(\alpha) C_{\lambda}^{*} \psi\right)=\left(R_{0}^{\perp}(\alpha)^{\frac{1}{2}} \mathcal{P}_{1}^{\perp} D^{*} \phi, R_{0}^{\perp}(\alpha)^{\frac{1}{2}} \mathcal{P}_{1}^{\perp} C_{\lambda} \psi\right) .
$$


To check boundedness of this form, it is therefore sufficient to verify that $R_{0}^{\perp}(\alpha)^{\frac{1}{2}} \mathcal{P}_{1}^{\perp} D^{*}$ and $R_{0}^{\perp}(\alpha)^{\frac{1}{2}} \mathcal{P}_{1}^{\perp} C_{\lambda}^{*}$ are bounded operators. We shall check it for their adjoints. To this purpose, it is enough to show that $C_{\lambda} \mathcal{P}_{1}^{\perp}$ and $D \mathcal{P}_{1}^{\perp}$ are $\left(R_{0}^{\perp}(\alpha)^{-\frac{1}{2}} \mathcal{P}_{1}^{\perp}\right)$-bounded, i.e., that there exist positive $a, b$ such that

$$
\forall \psi \in Q\left(H_{\lambda}\right): \quad\left\|C_{\lambda} \mathcal{P}_{1}^{\perp} \psi\right\| \leq a\left\|R_{0}^{\perp}(\alpha)^{-\frac{1}{2}} \mathcal{P}_{1}^{\perp} \psi\right\|+b\|\psi\|,
$$

and similarly for $D \mathcal{P}_{1}^{\perp}$. However,

$$
\begin{aligned}
\left\|\nabla \mathcal{P}_{1}^{\perp} \psi\right\|^{2} & =\left\|\left(H_{0}+1\right)^{\frac{1}{2}} \mathcal{P}_{1}^{\perp} \psi\right\|^{2}-\left\|\mathcal{P}_{1}^{\perp} \psi\right\|^{2} \\
\left\|\left(H_{0}+1\right)^{\frac{1}{2}} \mathcal{P}_{1}^{\perp} \psi\right\| & \leq\left\|\left(H_{0}-\alpha^{2}\right)^{\frac{1}{2}} \mathcal{P}_{1}^{\perp} \psi\right\|+\sqrt{1+\alpha^{2}}\left\|\mathcal{P}_{1}^{\perp} \psi\right\| \\
& \leq\left\|R_{0}^{\perp}(\alpha)^{-\frac{1}{2}} \mathcal{P}_{1}^{\perp} \psi\right\|+\sqrt{1+\alpha^{2}}\|\psi\| .
\end{aligned}
$$

Here $\nabla$ means the gradient in the variables $(x, u)$ ) through which all the actions of $C_{\lambda}, D$ can be estimated, e.g., $\left|\left(C_{\lambda} \psi\right)_{1}\right| \equiv\left|A_{1} \psi\right| \leq$ $2\|v\|_{\infty}|\nabla \psi|$, etc. In the same way, one verifies the analyticity of the operator-valued function $D R_{0}^{\perp}(\alpha) C_{\lambda}^{*}$, which is equivalent to the analyticity of the complex-valued function $\alpha \mapsto b_{\alpha}(\cdot, \cdot)$.

Consider next the regular part of $R_{0}(\alpha) \mathcal{P}_{1}$ containing the operator $N_{\alpha}$. Let $h$ be a $C^{\infty}$-function of compact support in $\mathbb{R}^{2}$. As pointed out above, using integration by parts and the explicit form of the operators $C_{\lambda}, D$ one sees that it is sufficient to check the boundedness and analyticity of $h n_{\alpha} h$ and $h n_{\alpha, \mu} h$, where

$$
\begin{aligned}
n_{\alpha}\left(x, x^{\prime}\right) & :=\frac{1}{2 \pi} K_{0}\left(k_{1}(\alpha)\left|x-x^{\prime}\right|\right)+\ln k_{1}(\alpha), \\
n_{\alpha, \mu}\left(x, x^{\prime}\right) & =-\frac{1}{2 \pi} \frac{x^{\mu}-x^{\prime \mu}}{\left|x-x^{\prime}\right|} k_{1}(\alpha) K_{1}\left(k_{1}(\alpha)\left|x-x^{\prime}\right|\right) ;
\end{aligned}
$$

recall that ${ }_{, \mu}$ means the derivative w.r.t. $x^{\mu}$ and $K_{0}^{\prime}=-K_{1}$ holds true - cf. [AS, 9.6.27]. We will use the following estimates which are valid for the Macdonald functions [AS, 9.6-7] with any $z \in(0, \infty)$ :

$$
\begin{aligned}
& \left|\left(K_{0}(z)+\ln z\right) e^{-z}\right| \leq c_{1}, \quad\left|K_{1}(z)-z^{-1}\right| \leq c_{2}, \\
& \left|\left[K_{1}(z)-z\left(K_{0}(z)+K_{2}(z)\right) / 2\right]\right| \leq c_{3}, \quad\left|z K_{1}(z)\right| \leq 1 .
\end{aligned}
$$


Passing to the polar coordinates,

$$
x^{\mu}-x^{\prime \mu}=(\rho \cos \varphi, \rho \sin \varphi), \quad \rho_{m}:=\sup _{x, x^{\prime} \in \operatorname{supp} h}\left|x-x^{\prime}\right|,
$$

we check the finiteness of the Schur-Holmgren bounds:

$$
\begin{aligned}
\left\|h n_{\alpha} h\right\|_{\mathrm{SH}} & =\sup _{x \in \mathbb{R}^{2}}|h(x)| \int_{\mathbb{R}^{2}}\left|m_{1}\left(x, x^{\prime} ; \alpha\right) h\left(x^{\prime}\right)\right| d x^{\prime} \\
& \leq c_{1}\|h\|_{\infty}^{2} \int_{0}^{\rho_{m}} e^{k_{1}(\alpha) \rho} \rho d \rho+\int_{0}^{\rho_{m}}|\ln \rho| \rho d \rho \\
& \leq c_{1}\|h\|_{\infty}^{2} \rho_{m}\left(\rho_{m} e^{\kappa_{1} \rho_{m}}+\max \left\{e^{-1}, \rho_{m} \ln \rho_{m}\right\}\right), \\
\left\|h n_{\alpha, \mu} h\right\|_{\mathrm{SH}} & \leq\|h\|_{\infty}^{2} \int_{0}^{\rho_{m}} \frac{\rho d \rho}{\rho}=\|h\|_{\infty}^{2} \rho_{m} .
\end{aligned}
$$

Concerning the analyticity, one should investigate the complex-valued functions $w \mapsto\left(\phi, h n_{\alpha(w)} h \psi\right)$ and $w \mapsto\left(\phi, h n_{\alpha(w), \mu} h \psi\right)$, where $\phi, \psi$ are arbitrary vectors of $\boldsymbol{L}^{2}\left(\Omega_{0}\right)$. Using the Schwarz inequality, it is sufficient to check the finiteness of norms of the complex derivative w.r.t. $w$ of the corresponding operator-valued functions. Since $K_{1}^{\prime}=$ $-\left(K_{0}+K_{2}\right) / 2$ by [AS, 9.6.29] and $k_{1}(\alpha(w))=e^{w^{-1}}$, we put $z:=$ $k_{1}(\alpha(w))\left|x-x^{\prime}\right|$ and write

$$
\begin{aligned}
\frac{d n_{\alpha(w)}}{d w}\left(x, x^{\prime}\right) & =\frac{1}{2 \pi} \frac{z}{w^{2}}\left(K_{1}(z)-\frac{1}{z}\right), \\
\frac{d n_{\alpha(w), \mu}}{d w}\left(x, x^{\prime}\right) & =\frac{1}{2 \pi} \frac{x^{\mu}-x^{\prime \mu}}{\left|x-x^{\prime}\right|} \frac{e^{w^{-1}}}{w^{2}}\left[K_{1}(z)-\frac{z}{2}\left(K_{0}(z)+K_{2}(z)\right)\right] .
\end{aligned}
$$

Using now the inequality $w^{-2} e^{w^{-1}} \leq c_{4}$ for $w \in(-\infty, 0)$, we are able to estimate the Schur-Holmgren bounds:

$$
\left\|h \frac{d n_{\alpha(w)}}{d w} h\right\|_{\mathrm{SH}} \leq c_{2} c_{4}\|h\|_{\infty}^{2} \rho_{m}^{2}, \quad\left\|h \frac{d n_{\alpha(w), \mu}}{d w} h\right\|_{\mathrm{SH}} \leq c_{3} c_{4}\|h\|_{\infty}^{2} \rho_{m}^{2} .
$$

Thus the derivatives are bounded for $w \in(-\infty, 0)$, and since the limits as $w$ tends to zero make sense, we can continue the function analytically to $w=0$. 
Now we are in position to follow the standard Birman-Schwinger scheme to derive the weak-coupling expansion. Eigenvalues of $H_{\lambda}$ correspond to singularities of the operator-valued function $\left(I+K_{\lambda}^{\alpha}\right)^{-1}$ which we can express as

$$
\left(I+K_{\lambda}^{\alpha}\right)^{-1}=\left[I+\left(I+\hat{M}_{\lambda}\right)^{-1} \hat{L}_{\lambda}\right]^{-1}\left(I+\hat{M}_{\lambda}\right)^{-1} .
$$

Owing to Lemma 2.3, $\left\|\hat{M}_{\lambda}\right\|$ is finite and we can choose $\lambda$ sufficiently small to have $\left\|\hat{M}_{\lambda}\right\|<1$; then the second term at the r.h.s. of (2.8) is a bounded operator. On the other hand, $\left(I+\hat{M}_{\lambda}\right)^{-1} \hat{L}_{\lambda}$ is a rank-one operator of the form $(\psi, \cdot) \varphi$, where

$$
\begin{aligned}
\bar{\psi}(x, u) & :=-\frac{\lambda}{2 \pi} \ln k_{1}(\alpha) \chi_{1}(u) C_{\lambda}^{*}, \\
\varphi(x, u) & :=\left[\left(I+\hat{M}_{\lambda}\right)^{-1} D \chi_{1}\right](x, u),
\end{aligned}
$$

so it has just one eigenvalue which is

$$
(\psi, \varphi)=-\frac{\lambda}{2 \pi} \ln k_{1}(\alpha) \int_{0}^{d} \int_{\mathbb{R}^{2}} \chi_{1}(u) C_{\lambda}^{*}\left[\left(I+\hat{M}_{\lambda}\right)^{-1} D \chi_{1}\right](x, u) d x d u
$$

Putting it equal -1 we get an implicit equation, $F(\lambda, w)=0$, with

$$
F(\lambda, w):=w-\frac{\lambda}{2 \pi} \int_{0}^{d} \int_{\mathbb{R}^{2}} \chi_{1}(u) C_{\lambda}^{*}\left[\left(I+\hat{M}_{\lambda}\right)^{-1} D \chi_{1}\right](x, u) d x d u,
$$

where $\hat{M}_{\lambda}$ has to be understood as a function both of $\lambda$ and $w$. Expanding $\left(I+\hat{M}_{\lambda}\right)^{-1}$ into the Neumann series we find

$$
F_{, w}(0,0)=1 \neq 0, \quad F_{, \lambda}(0,0)=-\frac{1}{2 \pi}\left(\chi_{1}, C_{0}^{*} D \chi_{1}\right),
$$

and by Lemma 2.3 we know that $F(\lambda, w)$ is jointly analytic in $\lambda, w$. In view of the implicit function theorem $w=w(\lambda)$ is then an analytic function and we can compute the first term in its Taylor expansion:

$$
\frac{d w}{d \lambda}(0)=-\frac{F_{, \lambda}(0,0)}{F_{, w}(0,0)}=\frac{1}{2 \pi}\left(\chi_{1}, C_{0}^{*} D \chi_{1}\right) .
$$


But $\left(C_{0}\right)_{n}=0$ for $n=4, \ldots, 7, B_{3} \chi_{1}=0$, and $\left(A_{2} \chi_{1}, B_{2} \chi_{1}\right)=0$ since $\int_{\mathbb{R}^{2}} \Delta v=0$. It follows that

$$
\frac{d w}{d \lambda}(0)=\frac{1}{2 \pi}\left(A_{1} \chi_{1}, B_{1} \chi_{1}\right)=-\frac{1}{\pi} \int_{0}^{d} \chi_{1}^{\prime}(u)^{2} d u \int_{\mathbb{R}^{2}} v(x) d x=-\frac{\kappa_{1}^{2}}{\pi}\langle v\rangle,
$$

where we have employed the symbol $\langle v\rangle:=\int_{\mathbb{R}^{2}} v(x) d x$.

We note that $\alpha^{2} \rightarrow \kappa_{1}^{2}$ - holds as $\lambda \rightarrow 0+$, and consequently, $k_{1}(\alpha) \rightarrow 0+$. Thus $w(0)=0$ is well defined because $w=\left(\ln k_{1}(\alpha)\right)^{-1}$ by definition. Furthermore, the solution $\alpha^{2}$ clearly represents an eigenvalue if and only if $w$ is strictly negative for $\lambda$ small. A sufficient condition for that is that the first term of the expansion of $w(\lambda)$ is strictly negative; due to (2.10) it happens if $\langle v\rangle$ is strictly positive. Summing up the discussion, we get the announced three-dimensional analogue to Theorem 1.2 in BGRS:

Theorem 2.4 Let $\Omega_{\lambda}$ be given by (2.1), where $v \in C_{0}^{\infty}\left(\mathbb{R}^{2}\right)$ satisfies $\langle v\rangle>0$. Then for all sufficiently small positive $\lambda,-\Delta_{D}^{\Omega_{\lambda}}$ has a unique eigenvalue $E(\lambda)$ in $\left[0, \kappa_{1}^{2}\right)$, which is simple and can be expressed as $E(\lambda)=\kappa_{1}^{2}-e^{2 w(\lambda)^{-1}}$, where $\lambda \mapsto w(\lambda)$ is an analytic function. Moreover, the following asymptotic expansion is valid:

$$
w(\lambda)=-\lambda \frac{\kappa_{1}^{2}}{\pi}\langle v\rangle+\mathcal{O}\left(\lambda^{2}\right) .
$$

\section{An alternative method}

Now we will derive the weak-coupling expansion by constructing the asymptotics for singularities in a particular boundary value problem. This approach enables us to derive easily higher terms of the expansion. At the same time it allows a unified treatment for different dimensions; in this way we will be able to amend the existing results concerning deformed strips.

First we introduce a unifying notation. Let $n=2,3$ be the dimension of the considered deformed region, i.e., the perturbed planar strip or layer, respectively. We set $x=\left(x^{1}, \ldots, x^{n-1}\right) \in \mathbb{R}^{n-1}$ 
and $(x, u) \in \Omega_{0}:=\mathbb{R}^{n-1} \times(0, d)$ for the unperturbed domain. From technical reasons it is convenient to change the setting slightly, in comparison with (2.1) and [BGRS], [EV3], and to deform the "lower" boundary of $\Omega_{0}$ what we certainly can do without loss of generality. We denote therefore in this section

$$
\Omega_{\lambda}:=\left\{(x, u) \in \mathbb{R}^{n}:-\lambda d v(x)<u<d\right\}
$$

with $v \in \boldsymbol{C}_{0}^{\infty}\left(\mathbb{R}^{n-1}\right)$. We denote by $-\Delta^{\prime}$ the $(n-1)$-dimensional Laplacian, while $-\Delta$ stands for the $n$-dimensional one. We also use

$$
\langle f\rangle:=\int_{\mathbb{R}^{n-1}} f(x) d x
$$

$\|\cdot\|$ as the norm in $\boldsymbol{L}^{2}\left(\mathbb{R}^{n-1}\right)$, and

$$
\alpha(m):=\left\{\begin{array}{ll}
m \\
(\ln m)^{-1}
\end{array} \quad \beta(t):= \begin{cases}t & \text { if } n=2 \\
\ln t & \text { if } n=3\end{cases}\right.
$$

\subsection{The asymptotic expansion}

Let us now construct the asymptotics of the eigenvalues $m_{\lambda}$ of the following boundary value problem:

$$
\begin{aligned}
& \left(\Delta+\kappa_{1}^{2}\right) \Psi_{\lambda}=m_{\lambda}^{2} \Psi_{\lambda} \quad \text { in } \Omega_{\lambda} \\
& \Psi_{\lambda}(x, \lambda d v(x))=\Psi_{\lambda}(x, d)=0
\end{aligned}
$$

as they approach zero. We will seek it in the form

$$
m_{\lambda}= \begin{cases}\sum_{i=1}^{\infty} \lambda^{i} m_{i} & \text { if } n=2 \\ \exp \left(-\left(\sum_{i=1}^{\infty} \lambda^{i} m_{i}\right)^{-1}\right) & \text { if } n=3\end{cases}
$$

where the existence of such expansions follows from BGRS and Theorem 2.4, respectively. Notice that this corresponds to the expansion of $E(\lambda)=\kappa_{1}^{2}-m_{\lambda}^{2}$, the ground-state eigenvalue of $-\Delta_{D}^{\Omega_{\lambda}}$ in the problem discussed above, because the mirror transformation of $\Omega_{\lambda}$ on (3.1) does not affect the spectral properties. 
Suppose that a function $f \in \boldsymbol{C}_{0}^{\infty}\left(\mathbb{R}^{n-1}\right)$, supp $f \cap \operatorname{supp} v=\emptyset$, and $\langle f\rangle \neq 0$ is given. If we manage to construct a solution $\psi_{\lambda}(x, u ; m)$ of the boundary value problem

$$
\begin{array}{r}
\left(\Delta+\kappa_{1}^{2}\right) \psi_{\lambda}=m^{2} \psi_{\lambda}+\left(\alpha(m)-\alpha\left(m_{\lambda}\right)\right) f \chi_{1} \quad \text { in } \quad \Omega_{\lambda} \\
\psi_{\lambda}=0 \text { on } \partial \Omega_{\lambda}
\end{array}
$$

which is bounded and non-vanishing w.r.t. $m$ for small nonzero $m$, then $\Psi_{\lambda}(x, u)=\psi_{\lambda}\left(x, u ; m_{\lambda}\right)$. We shall look for the asymptotics of $\psi_{\lambda}$ in the following form,

$$
\psi_{\lambda}(x, u ; m)=\sum_{i=0}^{\infty} \lambda^{i} \psi_{i}(x, u ; m) .
$$

Substituting (3.4) and (3.2) into (3.3), we obtain a family of the boundary value problems:

$$
\begin{array}{rlll}
\left(\Delta+\kappa_{1}^{2}\right) \psi_{0}=m^{2} \psi_{0}+\alpha(m) f \chi_{1} & \text { in } & \Omega_{0} & i=0 \\
\psi_{0}=0 & \text { on } & \partial \Omega_{0} & \\
\left(\Delta+\kappa_{1}^{2}\right) \psi_{i}=m^{2} \psi_{i}+(-1)^{n-1} m_{i} f \chi_{1} & \text { in } & \Omega_{0} & i \geq 1 \\
\psi_{i}=0 & \text { if } & u=d & \\
\psi_{i}=-\sum_{j=1}^{i} \frac{d^{j}(-v)^{j}}{j !} \frac{\partial^{j} \psi_{i-j}}{\partial u^{j}} & \text { if } & u=0
\end{array}
$$

One can check easily that $\psi_{0}=-\alpha(m)\left(-\Delta^{\prime}+m^{2}\right)^{-1} f \chi_{1}$ solves (3.5) and has the asymptotics

$$
\begin{aligned}
& \psi_{0}(x, u ; m)=\frac{(-1)^{n-1}}{2 \pi^{n-2}} \chi_{1}(u)[\langle f\rangle \\
& \quad+(-1)^{n-1} \alpha(m)\left(\int_{\mathbb{R}^{n-1}} \beta\left(\left|x-x^{\prime}\right|\right) f\left(x^{\prime}\right) d x^{\prime}+\delta_{n}^{3}(\gamma-\ln 2)\langle f\rangle\right) \\
& \left.\quad+\mathcal{O}\left(\alpha(m)^{2}\right)\right]
\end{aligned}
$$

as $m \rightarrow 0$, where $\gamma$ is the Euler number and $\delta_{n}^{j}$ the Kronecker delta. 
Lemma 3.1 Suppose that $F \in \boldsymbol{C}^{\infty}\left(\overline{\Omega_{0}}\right)$ with a bounded support and $H \in C_{0}^{\infty}\left(\mathbb{R}^{n-1}\right)$ have the expansions

$$
F(x, u ; m)=\sum_{i=0}^{\infty} \alpha(m)^{i} F_{i}(x, u), \quad H(x ; m)=\sum_{i=0}^{\infty} \alpha(m)^{i} H_{i}(x)
$$

as $m \rightarrow 0$. Define $F_{i, k}:=\int_{0}^{d} F_{i}(\cdot, u) \chi_{k}(u) d u$. Let $\phi_{0}$ be the solution of the boundary value problem

$$
\begin{array}{rll}
\left(\Delta+\kappa_{1}^{2}\right) \phi_{0}=F_{0} & \text { in } & \Omega_{0}, \\
\phi_{0}=0 & \text { if } & u=d \\
\phi_{0}=H_{0} & \text { if } & u=0
\end{array}
$$

then the condition

$$
\left\langle F_{0,1}\right\rangle=\sqrt{\frac{2}{d}} \kappa_{1}\left\langle H_{0}\right\rangle
$$

is necessary and sufficient for existence of a solution of the boundary value problem

$$
\begin{array}{rll}
\left(\Delta+\kappa_{1}^{2}\right) \phi=m^{2} \phi+F & \text { in } & \Omega_{0}, \\
\phi=0 & \text { if } & u=d, \\
\phi=H & \text { if } & u=0,
\end{array}
$$

which is bounded as $m \rightarrow 0$. If it is satisfied, the solution has the asymptotics

$$
\begin{aligned}
& \phi(x, u ; m) \\
& \quad=\phi_{0}(x, u)+\frac{(-1)^{n-1}}{2 \pi^{n-2}} \chi_{1}(u)\left(\left\langle F_{1,1}\right\rangle-\sqrt{\frac{2}{d}} \kappa_{1}\left\langle H_{1}\right\rangle\right)+\mathcal{O}(\alpha(m)) .
\end{aligned}
$$

Proof: The statement is obvious if $H=0$. In particular, the solution $\phi$ is constructed by the Fourier method in the explicit form

$$
\phi(x, u ; m)=\sum_{i=1}^{\infty} \tilde{\phi}_{i}(x ; m) \chi_{i}(u)
$$


By a direct calculation it is easy to see that $\tilde{\phi}_{i}$ are bounded functions for $m \geq 0$ so long as $i \geq 2$. The problem arises for $i=1$, because in general $\tilde{\phi}_{1}$ tends to infinity as $m \rightarrow 0$. The condition (3.9) guarantees that the explicit solution $\phi$ has no such pole. This proves the sufficiency. To see that the condition is necessary at the same time, one integrates by parts in the scalar product equation

$$
\left(\chi_{1},\left(\Delta+\kappa_{1}^{2}-m^{2}\right) \phi\right)=\left(\chi_{1}, F\right)
$$

and puts $m=0$ afterwards. In the opposite case, $H \neq 0$, we use the replacement

$$
\phi(x, u ; m)=\varphi(x, u ; m)+\left(1-\frac{u}{d}\right) H(x ; m)
$$

and expand the r.h.s. of the equation for $\varphi$ in the Fourier series, which reduces the task to the previous situation.

Corollary $3.2 \phi \in \boldsymbol{C}^{\infty}(\bar{Q})$ holds for any bounded domain $Q \subset \Omega_{0}$.

It follows from Lemma 3.1 that the recursive system of the boundary value problem (3.6) has solutions which are continuous with respect to $m$ in the vicinity of $m=0$ and decay as $|x| \rightarrow \infty$ for $m>0$, provided the $m_{i}$ 's satisfy the following recursive relations:

$$
m_{i}=(-1)^{n} \sqrt{\frac{2}{d}} \frac{\kappa_{1}}{\langle f\rangle} \sum_{j=1}^{i}\left\langle\frac{d^{j}(-v)^{j}}{j !} \frac{\partial^{j} \psi_{i-j}}{\partial u^{j}}(\cdot, 0 ; 0)\right\rangle .
$$

In particular, owing to (3.7) and Lemma 3.1 we get

$$
m_{1}=\frac{\kappa_{1}^{2}}{\pi^{n-2}}\langle v\rangle
$$

which agrees with the leading term obtained by the Birman-Schwinger method in the previous section - cf. Theorem 2.4 and (3.2) - as well as with the corresponding result (1.1) in the strip case. 


\subsection{The next-to-leading order}

Let us now calculate $m_{2}$. By virtue of (3.6), (3.7) and (3.11) the boundary value problem for $\psi_{1}$ together with the boundary condition for $\psi_{2}(x, u ; 0)$ look as follows

$$
\begin{aligned}
&\left(\Delta+\kappa_{1}^{2}\right) \psi_{1}=m^{2} \psi_{1}+(-1)^{n-1} \frac{\kappa_{1}^{2}}{\pi^{n-2}}\langle v\rangle f \chi_{1} \text { in } \quad \Omega_{0} \\
& \psi_{1}=0 \text { if } \quad u=d \\
& \psi_{1}=d v \frac{\partial \psi_{0}}{\partial u} \text { if } \quad u=0 \\
& \psi_{2}=0 \text { if } \quad u=d \\
& \psi_{2}=d v \frac{\partial \psi_{1}}{\partial u} \quad \text { if } \quad u=d, m=0
\end{aligned}
$$

with

$$
\frac{\partial \psi_{0}}{\partial u}(x, 0 ; m)=\frac{(-1)^{n-1}}{2 \pi^{n-2}} \sqrt{\frac{2}{d}} \kappa_{1} \mathcal{B}(f),
$$

where $\mathcal{B}(f)$ is the square bracket from (3.7). Hence

$$
m_{2}=(-1)^{n-1} \sqrt{\frac{2}{d}} \frac{\kappa_{1} d}{\langle f\rangle}\left\langle v \frac{\partial \psi_{1}}{\partial u}(\cdot, 0 ; 0)\right\rangle
$$

and it is sufficient to find $\psi_{1}$. With eq. (3.12) and Lemma 3.1 in mind, we consider the following boundary value problem

$$
\begin{aligned}
\left(\Delta+\kappa_{1}^{2}\right) \phi_{0}=(-1)^{n-1} \frac{\kappa_{1}^{2}}{\pi^{n-2}}\langle v\rangle f \chi_{1} \quad \text { in } \quad \Omega_{0} \\
\phi_{0}=0 \quad \text { if } \quad u=d \\
\phi_{0}=\frac{(-1)^{n-1}}{2 \pi^{n-2}} \sqrt{\frac{2}{d}} \kappa_{1} d v\langle f\rangle \quad \text { if } \quad u=0
\end{aligned}
$$

and seek $\phi_{0}$ in the form

$$
\phi_{0}(x, u)=\frac{(-1)^{n-1}}{2 \pi^{n-2}} \sqrt{\frac{2}{d}} \kappa_{1}\left[\left(1-\frac{u}{d}\right)\langle f\rangle d v(x)-\varphi(x, u)\right]
$$


substituting it into (3.15), we arrive at the boundary value problem

$$
\begin{array}{r}
\left(\Delta+\kappa_{1}^{2}\right) \varphi=-d\langle f\rangle\left(1-\frac{u}{d}\right)\left(\Delta^{\prime}+\kappa_{1}^{2}\right) v+2 \kappa_{1} \sqrt{\frac{2}{d}}\langle v\rangle f \chi_{1} \quad \text { in } \quad \Omega_{0} \\
\varphi=0 \quad \text { on } \partial \Omega_{0} .
\end{array}
$$

The Fourier method gives

$$
\begin{aligned}
\varphi= & -\sqrt{\frac{2}{d}} d\langle f\rangle \sum_{k=2}^{\infty} \frac{\chi_{k}}{\kappa_{k}}\left(-\Delta^{\prime}+\kappa_{k}^{2}-\kappa_{1}^{2}\right)^{-1}\left(-\Delta^{\prime}-\kappa_{1}^{2}\right) v \\
& -\sqrt{\frac{2}{d}} d \frac{\chi_{1}}{\kappa_{1}}\left[\langle f\rangle v+\kappa_{1}^{2}\left(-\Delta^{\prime}\right)^{-1}(\langle v\rangle f-\langle f\rangle v)\right] .
\end{aligned}
$$

Lemma 3.1 an relations (3.12), (3.13), (3.15), and (3.16) together with the last result imply that

$$
\begin{aligned}
& \frac{\partial \psi_{1}}{\partial u}(x, 0 ; 0)=\frac{(-1)^{n}}{2 \pi^{n-2}} \sqrt{\frac{2}{d}} \kappa_{1} \\
& \quad \times\left\{\frac { \kappa _ { 1 } ^ { 2 } } { \pi ^ { n - 2 } } \left[\int_{\mathbb{R}^{n-1} \times \mathbb{R}^{n-1}} v(x) \beta\left(\left|x-x^{\prime}\right|\right) f\left(x^{\prime}\right) d x d x^{\prime}\right.\right. \\
& \left.\quad+\langle f\rangle \int_{\mathbb{R}^{n-1}} \beta\left(\left|x-x^{\prime}\right|\right) v\left(x^{\prime}\right) d x^{\prime}-\langle v\rangle \int_{\mathbb{R}^{n-1}} \beta\left(\left|x-x^{\prime}\right|\right) f\left(x^{\prime}\right) d x^{\prime}\right] \\
& \quad+\langle f\rangle\left[3 v(x)+2 \sum_{k=2}^{\infty}\left[\left(-\Delta^{\prime}+\kappa_{k}^{2}-\kappa_{1}^{2}\right)^{-1}\left(-\Delta^{\prime}-\kappa_{1}^{2}\right) v\right](x)\right. \\
& \left.\left.\quad+\delta_{n}^{3} \frac{\kappa_{1}^{2}}{\pi}(\gamma-\ln 2)\langle v\rangle\right]\right\},
\end{aligned}
$$

where we have employed also the implication

$$
\langle F\rangle=0 \Rightarrow\left(-\Delta^{\prime}\right)^{-1} F=\frac{-1}{2 \pi^{n-2}} \int_{\mathbb{R}^{n-1}} \beta\left(\left|\cdot-x^{\prime}\right|\right) F\left(x^{\prime}\right) d x^{\prime} .
$$


Substituting this into (3.14) we get the sought coefficient:

$$
\begin{aligned}
m_{2}= & -\frac{\kappa_{1}^{2}}{\pi^{n-2}}\left\{3\left\langle v^{2}\right\rangle+\frac{\kappa_{1}^{2}}{\pi^{n-2}} \int_{\mathbb{R}^{n-1} \times \mathbb{R}^{n-1}} v(x) \beta\left(\left|x-x^{\prime}\right|\right) v\left(x^{\prime}\right) d x d x^{\prime}\right. \\
& +2\left\langle v \sum_{k=2}^{\infty}\left(-\Delta^{\prime}+\kappa_{k}^{2}-\kappa_{1}^{2}\right)^{-1}\left(-\Delta^{\prime}-\kappa_{1}^{2}\right) v\right\rangle \\
& \left.+\delta_{n}^{3} \frac{\kappa_{1}^{2}}{\pi}(\gamma-\ln 2)\langle v\rangle^{2}\right\}
\end{aligned}
$$

\subsection{The critical case}

As we have pointed out in the introduction, the above result is most interesting in the critical case, $\langle v\rangle=0$, when the first coefficient (3.11) equals zero and $m_{2}$ given by (3.17) determines the leading order. In this situation we have the following result.

Theorem 3.3 Let $V \in C_{0}^{\infty}\left(\mathbb{R}^{n-1}\right)$ be an arbitrary function such that $\langle V\rangle=0$ and

$$
v(x)=V\left(\frac{x}{\sigma}\right), \quad \sigma>0 .
$$

Then the following inequalities hold,

$$
\begin{array}{r}
-\frac{\kappa_{1}^{2} \sigma^{n-1}}{\pi^{n-2}}\left(\frac{8}{2}\|V\|^{2}+\frac{3}{2 \kappa_{1}^{2} \sigma^{2}}\|V\|\left\|\Delta^{\prime} V\right\|-2 \kappa_{1}^{2} \sigma^{2}\left\|\nabla^{\prime}\left(\Delta^{\prime}\right)^{-1} V\right\|^{2}\right) \\
\leq m_{2} \leq-\frac{\kappa_{1}^{2} \sigma^{n-1}}{\pi^{n-2}}\left(\frac{3}{2}\|V\|^{2}-2 \kappa_{1}^{2} \sigma^{2}\left\|\nabla^{\prime}\left(\Delta^{\prime}\right)^{-1} V\right\|^{2}\right) .
\end{array}
$$

ProOF: In the first place, note that $\langle V\rangle=0$ implies

$$
-\frac{\kappa_{1}^{2}}{\pi^{n-2}} \int_{\mathbb{R}^{n-1}} V(x) \beta\left(\left|x-x^{\prime}\right|\right) V\left(x^{\prime}\right) d x d x^{\prime}=\left\|\nabla^{\prime}\left(\Delta^{\prime}\right)^{-1} V\right\|^{2}>0,
$$

because $\Delta^{\prime} \beta(|x|)=2 \pi^{n-2} \delta(x)$ holds in the sense of distribution. Under the stated assumptions, the formula (3.17) yields therefore

$$
m_{2}=-\frac{\kappa_{1}^{2} \sigma^{n-1}}{\pi^{n-2}}\left(3\|V\|^{2}-2 \kappa_{1}^{2} \sigma^{2}\left\|\nabla^{\prime}\left(\Delta^{\prime}\right)^{-1} V\right\|^{2}+2 A(\sigma)\right)
$$


where

$$
A(\sigma):=\sum_{k=2}^{\infty}\left\langle V\left(-\Delta^{\prime}+\left(\kappa_{k}^{2}-\kappa_{1}^{2}\right) \sigma^{2}\right)^{-1}\left(-\Delta^{\prime}-\kappa_{1}^{2} \sigma^{2}\right) V\right\rangle,
$$

and it suffices to find suitable bounds on $A(\sigma)$.

Since the Fourier transformation together with the Plancherel theorem give the estimate

$$
\left\|\left(-\Delta^{\prime}+\left(\kappa_{k}^{2}-\kappa_{1}^{2}\right) \sigma^{2}\right)^{-1} F\right\| \leq \frac{\|F\|}{\left(\kappa_{k}^{2}-\kappa_{1}^{2}\right) \sigma^{2}},
$$

we obtain the upper bound

$$
A(\sigma) \leq \frac{3}{4}\left(\|V\|^{2}+\frac{1}{\kappa_{1}^{2} \sigma^{2}}\|V\|\left\|\Delta^{\prime} V\right\|\right),
$$

where the numerical factor comes from $\sum_{k=2}^{\infty}\left(k^{2}-1\right)^{-1}=\frac{3}{4}$.

On the other hand, denoting

$$
U_{k}(x ; \sigma):=\left[\left(-\Delta^{\prime}+\left(\kappa_{k}^{2}-\kappa_{1}^{2}\right) \sigma^{2}\right)^{-1} V\right](x),
$$

we see that

$$
\begin{aligned}
& \left\langle V\left(-\Delta^{\prime}+\left(\kappa_{k}^{2}-\kappa_{1}^{2}\right) \sigma^{2}\right)^{-1}\left(-\Delta^{\prime}-\kappa_{1}^{2} \sigma^{2}\right) V\right\rangle \\
& \quad=\left\langle\left(-\Delta^{\prime}+\left(\kappa_{k}^{2}-\kappa_{1}^{2}\right) \sigma^{2}\right) U_{k}\left(-\Delta^{\prime}-\kappa_{1}^{2} \sigma^{2}\right) U_{k}\right\rangle .
\end{aligned}
$$

Integrating the r.h.s. by parts and using (3.18), we get the lower bound

$$
\begin{aligned}
A(\sigma) & =\sum_{k=2}^{\infty}\left(\left\|\Delta^{\prime} U_{k}\right\|^{2}+\kappa_{1}^{2}\left(k^{2}-2\right) \sigma^{2}\left\|\nabla^{\prime} U_{k}\right\|^{2}-\kappa_{1}^{4}\left(k^{2}-1\right) \sigma^{4}\left\|U_{k}\right\|^{2}\right) \\
& >-\sum_{k=2}^{\infty} \kappa_{1}^{4}\left(k^{2}-1\right) \sigma^{4}\left\|U_{k}\right\|^{2} \geq-\|V\|^{2} \sum_{k=2}^{\infty} \frac{1}{k^{2}-1}=-\frac{3}{4}\|V\|^{2},
\end{aligned}
$$

which concludes the proof.

This theorem confirms the spectral picture we got from (1.2) and (1.3). More specifically, $m_{2}>0$ as $\sigma \rightarrow \infty$ so the critical weakly bound state exists for sufficiently smeared deformations, and vice versa. In contrast to (1.2) and (1.3), however, we are able now to tell from (3.17) for any given zero-mean $v$ the sign of $m_{2}$. 


\section{Acknowledgment}

R.G. is grateful for the hospitality extended to him at NPI AS where a part of this work was done. The research has been partially supported by GA AS and the Czech Ministry of Education under the contracts 1048801 and ME170. The first and the third authors have been partially supported by Russian Fund of Basic Research - Grants 99-01-00139 and 99-01-01143, respectively.

\section{References}

[AS] M.S. Abramowitz, I.A. Stegun, eds., Handbook of mathematical functions, Dover, New York 1965.

[AGH] S. Albeverio, F. Gesztesy, R. Høegh-Krohn, H. Holden, Solvable models in quantum mechanics, Springer, Heidelberg 1988.

[BGRS] W. Bulla, F. Gesztesy, W. Renger, B. Simon, Weakly coupled bound states in quantum waveguides, Proc. Amer. Math. Soc. 127 (1997), 1487-1495.

[DE] P. Duclos, P. Exner: Curvature-induced bound states in quantum waveguides in two and three dimensions, Rev. Math. Phys. 7 (1995), 73-102.

[DEK] P. Duclos, P. Exner, D. Krejčiřík: Locally curved quantum layers, Ukrainian J. Phys. 45 (2000), 595-601.

[EK] P. Exner, D. Krejčiřík: Waveguides coupled through a semitransparent barrier: a Birman-Schwinger analysis, Rev. Math. Phys. (2000), to appear; preprint mp_arc 00-248.

[EV1] P. Exner, S.A. Vugalter: Asymptotic estimates for bound states in quantum waveguides coupled laterally through a narrow window, Ann. Inst. H. Poincaré: Phys. théor. 65, 109-123 (1996).

[EV2] P. Exner, S.A. Vugalter: Bound-state asymptotic estimates for window-coupled Dirichlet strips and layers, J. Phys. A30 (1997), 7863-7878.

[EV3] P. Exner, S.A. Vugalter: Bound states in a locally deformed waveguide: the critical case, Lett. Math. Phys. 39 (1997), 59-68. 
[Ga] R.R. Gadyl'shin: Surface potentials and the method of matching asymptotic expansions in the problem of the Helmholtz resonator, Algebra i Analiz 4 (1992), 88-115; English transl. in St. Peterburgs Math. J. 4 (1993), 273-296.

[Il] A.M. Il'in: Matching of Asymptotic Expansions of Solutions of Boundary Value Problems, Nauka, Moscow 1989; English transl., Amer. Mat. Soc., Providence, RI, 1992.

[Mad] I.J. Maddox: Elements of Functional Analysis, Cambridge Univ. Press 1970.

[Po] I.Yu. Popov: Asymptotics for bound state for laterally coupled waveguides, Rep. Math. Phys. 4 (1992), 88-115.

[RS] M. Reed, B. Simon, Methods of modern mathematical physics, IV. Analysis of operators, Academic Press, New York 1978.

R. Borisov and R. Gadyl'shin

Bashkir State Pedagogical University

October Revolution St. 3a

RU-450000 Ufa, Russia

Email: borisovDI@ic.bashedu.ru, gadylshin@bspu.ru

P. Exner and D. Krejčiřík

Department of Theoretical Physics

Nuclear Physics Institute

Academy of Sciences

CZ-25068 Rež, Czech Republic

Email: exner@ujf.cas.cz, krejcirik@ujf.cas.cz 